\title{
CONVENTIONAL PCR BASED DETECTION OF Brucella abortus INFECTED CATTLE IN SOME SELECTED AREAS OF BANGLADESH
}

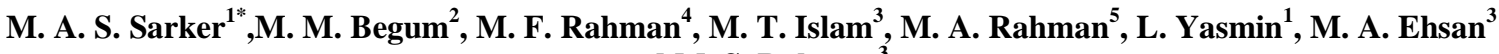 \\ and M. S. Rahman ${ }^{3}$ \\ ${ }^{1}$ Department of Livestock Services, Dhaka, Bangladesh $;{ }^{2}$ Youth Training Centre, Munshigonj, Ministry of Youth \\ and Sports, Dhaka, Bangladesh; ${ }^{3}$ Department of Medicine, Bangladesh Agricultural University, Mymensingh- \\ 2202, Bangladesh ; ${ }^{4}$ Commandant Armed Forces Medical College, Dhaka Cantonment, Dhaka ${ }^{5}$ Department of \\ Surgery and Theriogenology, Jhenidah Government Veterinary College, Jhenidah, Bangladesh
}

\begin{abstract}
Brucella spp. are facultative intracellular bacteria causing chronic disease which may persist for the whole life of the affected organism. In animals brucellosis affects reproduction, fertility and reduces newborns survival and also milk production. The present research was carried out to estimate the prevalence and to identify the risk factors of brucellosis along with determination of genetic diversity in Bangladesh. In CCBS\&DF (government farm) out of 191 cows the MRT prevalence (positive) was $7.85 \%$ and RBT prevalence (positive) was $7.33 \%$. In Rangpur out of 238 cows the MRT prevalence (positive) was $1.88 \%$ and RBT prevalence is $1.56 \%$. In Jamalpur out of 201 cows the MRT prevalence was $1.49 \%$ and RBT prevalence $0.05 \%$. In Gaibandha out of 93 cows the MRT prevalence was $1.07 \%$ and RBT prevalence $0 \%$. In Mymensingh out of 320 cows the MRT prevalence was $1.88 \%$ and RBT prevalence $1.56 \%$. Among the five groups of cows the high prevalence of MRT and RBT prevalence was in Government Farm $7.85 \%$ and $7.33 \%$. On the other hand the lower prevalence in cows of Gaibandha which was MRT and RBT $1.07 \%$ and $0 \%$ respectively and followed by Jamalpur MRT $1.49 \%$ and RBT $0.50 \%$, in Rangpur MRT 1.68\% and RBT 1.26\%, and in Mymensingh district MRT 1.88\% and RBT 1.56\%. The prevalence of brucellosis was significantly ( $\mathrm{p}<0.01$ ) higher in CCBS\&DF than all district (Table 8). Out of 14 MRT and RBT positive milk samples of CCBS\&DF (21.43\%) were PCR positive but all other 9 such samples originated from Jamalpur, Rangpur, Gaibandha and Mymensingh districts were PCR negative.
\end{abstract}

Keywords: cattle, MRT, RBT, PCR, prevalence, area, brucellosis

\section{INTRODUCTION}

Brucellosis is a recognized public health problem with worldwide distribution and one of the major causes of morbidity. Brucellosis causes a great economic loss to the livestock industries through abortion, infertility, birth of weak dead offspring, increased calving interval and reduction of milk yield and it is endemic in Bangladesh (Rahman et al., 2014). In order to control and eradicate brucellosis from human and livestock, it is very essential to establish an appropriate serological method for diagnosis of brucellosis in endemic areas. The geological distribution of brucellosis is constantly changing, with new foci emerging or re-emerging. Direct person to person spread of brucellosis is extremely rare. Mothers those who are breast feeding may transmit the disease or infection to their infants and sexual transmission has also been reported (Carrera et al., 2006; Kato et al., 2007). In countries where milk and dairy products are always pasteurized before consumption brucellosis mainly affects persons who are in close contact with animals and animal products. Prevalence of brucellosis in cattle might constitute a significant hurdle for the development of livestock in Bangladesh. So, early and accurate diagnosis is important for effective control measure against brucellosis. The MRT and RBT are widely used for screening of brucellosis exclusively in eradication programs. The RBT is a simple agglutination technique. These tests were chosen because they are less cumbersome to perform on a large scale and/or require no special equipment and expertise compared with other commonly used assays such as CFT and ELISA. Therefore, the present study was done to know the area wise prevalence of brucellosis in cattle and conventional PCR based detection of Brucella abortus in organized and smallholder dairy farms in some selected areas of Bangladesh.

*Corresponding e-mail address: sayeedsarker68@gmail.com 
Sarker and Others

\section{MATERIALS AND METHODS}

The molecular tests were performed like conventional PCR at Department of Microbiology and Hygiene, Bangladesh Agricultural University, Mymensingh.DNA extracted from milk samples by using Wizard Genomic DNA Purification Kit (Promega, USA) according to manufacturer's instruction and then PCR was performed. Briefly, at first, the required number of PCR tubes were labeled and kept on ice. Then $23 \mu 1$ of reaction mixture was dispensed into each of the PCR tubes and $2 \mu \mathrm{l}$ of DNA template from each sample was added to that respective tube and mixed well with the help of the micropipette. The tubes were placed in a twenty-four wells thermo cycler (Eppendorf, Germany). Then the temperature of the thermo cycler was set according to the thermal profile mentioned below. After completion of PCR, PCR products were separated by electrophoresis in a (1X TAE) $1 \%$ agarose gel stained by ethidium bromide. The band was then visualized with a medium wavelength UV light. Primer sequence of alkB genes, reaction and PCR assay conditions were given in Tables 2, 3, 4 .

Table 1.Reaction mixture used in PCR.

\begin{tabular}{ll}
\hline Composition & Amount $(\mu 1)$ \\
\hline Master mixture 2x (Promega, USA) & 12.5 \\
Genomic DNA( Template) & 2.0 \\
Primer (F) & 1.0 \\
Primer ( R) & 1.0 \\
Nuclease free water & 8.5 \\
\hline Total & 25.0 \\
\hline
\end{tabular}

*Note: prepared reaction mixer on $4^{0} \mathrm{C}$ PCR cooler

Table 2. Polymerase Chain Reaction conditions for alkB genes.

\begin{tabular}{lll}
\hline Cycling parameters & Temperature & Duration \\
Initial denaturation & $95^{\circ} \mathrm{c}$ & 10 minutes \\
Denaturation & $94^{\circ} \mathrm{c}$ & 15 seconds \\
Annealing & $54^{\circ} \mathrm{c}$ & 1 minute \\
Extension & $72^{\circ} \mathrm{c}$ & 1 minute \\
Final extension & $72^{\circ} \mathrm{c}$ and 40 cycles & 10 minutes \\
\hline
\end{tabular}

Table 3. Sequence of primers for alkB genes.

\begin{tabular}{lll}
\hline Primers(F) & 5'-GCGGCTTTTCTACACGGTATTC-3' & Terziet al(2010) \\
Primers(R) & 5'-CATGCGCTATGATCTGGTTACG-3' & \\
\hline
\end{tabular}

In this study required clinical, epidemiological, environmental and reproductive information recorded. During the study period a questionnaire based data on age sex, geographical area, status of pregnancy, disease history, hygroma, reproductive disorder such as abnormal abdominal uterine discharge, abortion, retention of placenta, and reproductive diseases were recorded. The RBT was used as a screening test to identify the infected animal and human with Brucella abortus strain1119-3 (Dae Sung Microbiological lab, South Korea). iELISA (Svanova Biotech AB, Uppsala Sweden) RBT and SAT were performed according to the procedure described by OIE (2009). The RBT positive sera were re-tested with SAT CFT, ELISA and qRT-PCR. For the qRT-PCR, DNA was isolated from $200 \mu \mathrm{L}$ of seropositive serum using the high pure PCR template Preparation Kit (Roche Diagnostic, Mannheim, Germany) according to manufacturer's instructions. Brucella IS711 targeting genus specific qRT-PCR was done according to the established and routine protocol (Tomaso et al., 2010) on a light cycler 2.0 instrument (Roche, Mannheim, Germany). Cycler threshold values (CT) $\leq 40$ were interpreted as positive. Positive samples were then typed with the Brucella IS711 species specific qRT-PCRs for Brucella abortus and Brucella melitensis according to Probert et al. (2004). CT values were calculated by the instrument's software MxPro3000P v 4.01. CT values $\leq 42$ were interpreted as positive. iELISA and CFT were performed according to protocol provided by the iELISA and CFT kits manufacturer company.

Data analysis: The Chi-square test $\left(\chi^{2}\right)$ was performed to find out the relationship between the prevalence of brucellosis and demographic variables of cows. The questionnaire based data was processed by Microsoft Excel and MSTATC, the results were statistically analyzed for interpretation by using Chi-square tests. Significance was determined at 1 to $5 \%$ level where applicable. 


\section{RESULTS}

In CCBS\&DF (government farm) out of 191 cows the MRT prevalence (positive) was $7.85 \%$ and RBT prevalence (positive) was $7.33 \%$. In Rangpur out of 238 cows the MRT prevalence (positive) was $1.88 \%$ and RBT prevalence is $1.56 \%$. In Jamalpur out of 201 cows the MRT prevalence was $1.49 \%$ and RBT prevalence $0.05 \%$. In Gaibandha out of 93 cows the MRT prevalence was $1.07 \%$ and RBT prevalence $0 \%$. In Mymensingh out of 320 cows the MRT prevalence was $1.88 \%$ and RBT prevalence $1.56 \%$. Among the five groups of cows the high prevalence of MRT and RBT prevalence was in Government Farm 7.85\% and 7.33\%. On the other hand the lower prevalence in cows of Gaibandha which was MRT and RBT 1.07\% and 0\% respectively and followed by Jamalpur MRT 1.49\% and RBT 0.50\%, in Rangpur MRT 1.68\% and RBT 1.26\%, and in Mymensingh district MRT $1.88 \%$ and RBT $1.56 \%$.

Table 4.Area-wise prevalence of brucellosis in cattle based on MRT and RBT.

\begin{tabular}{llllllll}
\hline Area & $\begin{array}{l}\text { No. of cows } \\
\text { tested }\end{array}$ & $\begin{array}{l}\text { MRT } \\
\text { positive }\end{array}$ & $\begin{array}{l}\text { Prevalence } \\
\text { on MRT \% }\end{array}$ & $\begin{array}{l}\text { RBT } \\
\text { positive }\end{array}$ & $\begin{array}{l}\text { Prevalence on } \\
\text { RBT \% }\end{array}$ & $\begin{array}{l}\text { Level } \\
\text { significance }\end{array}$ \\
\hline CCBS\&DF & 191 & 15 & 7.85 & 14 & 7.33 & & \\
Mymensingh & 320 & 6 & 1.88 & 5 & 1.56 & \multirow{2}{*}{ of } \\
Rangpur & 238 & 4 & 1.68 & 3 & 1.26 & \\
Jamalpur & 201 & 2 & 1.49 & 1 & 0.50 & & \\
Gaibandha & 93 & 1 & 1.07 & 0 & 0 & \\
\hline Total & 1043 & 28 & 2.68 & 23 & 2.21 & & \\
\hline
\end{tabular}

** Significant at $\mathrm{p} \leq 0.01$

Table 5. Comparative analysis between MRT, RBT and PCR results

\begin{tabular}{llll}
\hline MRT and RBT positive & Tested & PCR positive & Prevalence $(\%)$ \\
\hline CCBDF & 14 & 3 & 21.43 \\
Jamalpur and Rangpur districts & 4 & 0 & 0 \\
Gaibandha and Mymensingh districts & 5 & 0 & 0 \\
\hline
\end{tabular}

The prevalence of brucellosis was significantly $(\mathrm{p}<0.01)$ higher in CCBS\&DF than all district (Table 8). Out of 14 MRT and RBT positive milk samples of CCBS\&DF (21.43\%) were PCR positive but all other 9 such samples originated from Jamalpur, Rangpur, Gaibandha and Mymensingh districts were PCR negative (Table 5).

\section{DISCUSSION}

Brucellosis is an important zoonosis and serological surveillance is essential for its control (Erdenebaatar et al., 2004).The importance of brucellosis was primarily due to public health significance and economic loss. Bangladesh has been reported as an endemic area for brucellosis due to a considerable number of human and animal populations are exposed to infection every year (Nahar and Ahmed, 2009; Ahasan et al., 2010; Rahman et al., 2011). RBT is used as a screening test of Brucella infection (MacMillan, 1990) and it is reported to be more sensitive than the CFT in case of culture positive animal (Blasco et al., 1994). The overall prevalence of brucellosis based on MRT and RBT was recorded as $2.68 \%$ and $2.21 \%$ respectively. The MRT is generally used for screening; other test are required for confirmatory diagnosis (Sarker et al., $2014 \mathrm{a}$, b).This is an agreement with Morgan(1967) who stated that the test should be used in conjunction with the established tests and not instead of them . The higher proportion of positives by MRT might result from false positives which could be due to many causes including mastitis, colostrums, collection at the end of lactation period or a hormonal disorder (Bercovich and Moerman, 1979).Carefully samples were collected from dairy cows excluding mastitis infected and recently delivered cows to avoid false positive reactions. It has been shown that different serological tests used for the diagnosis of brucellosis vary considerably in their ability to detect antibodies of a particular immunoglobulin class. Infected animals may or may not produce all antibody isotypes in detectable quantities (FAO, 2004). Vaccination against brucellosis is not carried out in Bangladesh, so that our result may reflect natural infection. In general the MRT have been shown in other studies to have high sensitivity but lower specificity. The MRT is not normally used on individual animals because of false positives (less 
Sarker and Others

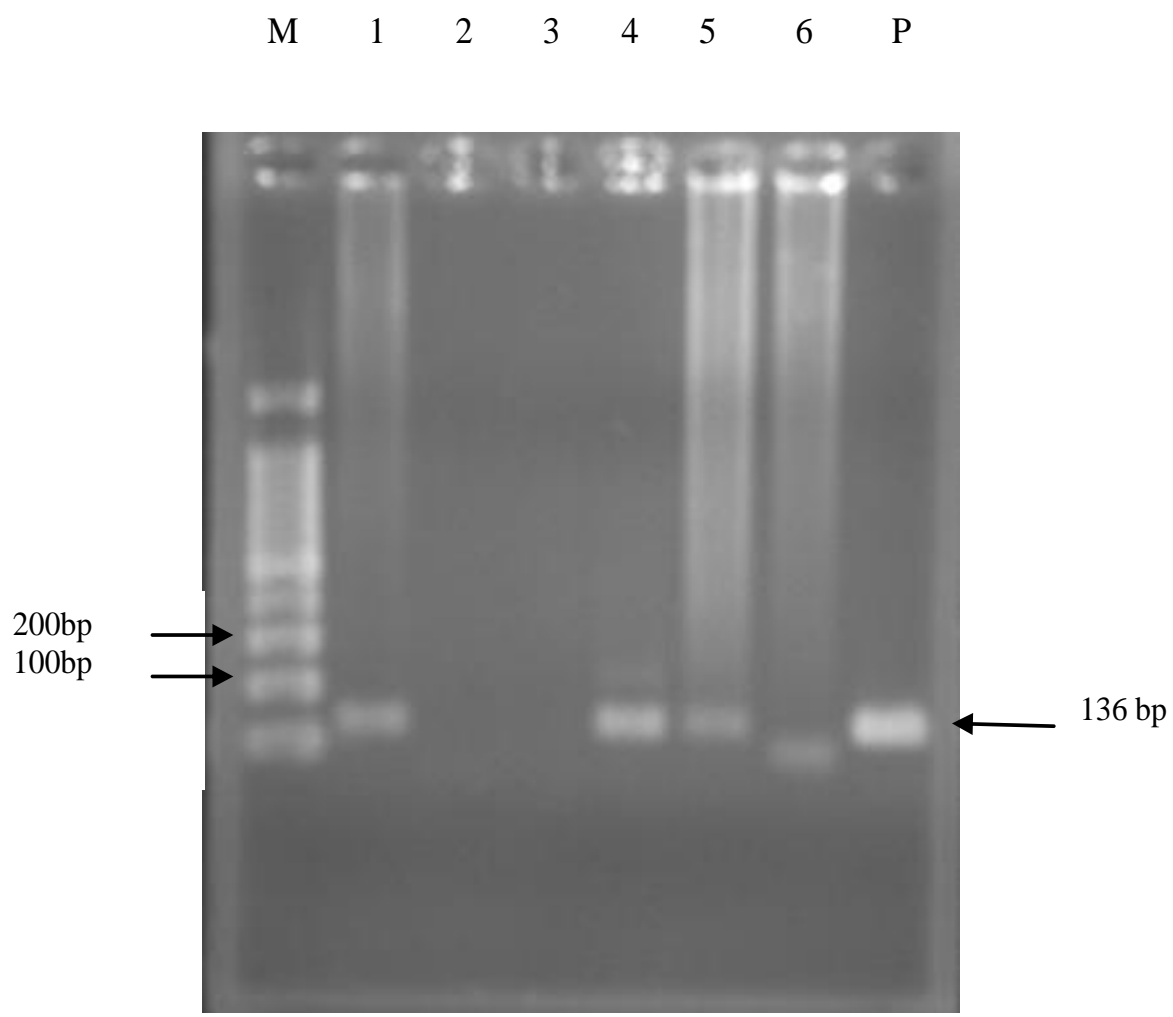

Figure 31. PCR for detection B.abortus from MRT positive milk samples. M- DNA marker (100bp ladder), L1 - 6 samples, LP -Positive control. PCR amplicon is analised in 2\% agarose and visualized by

specificity). Based on outcome of the study, it is suggested that although MRT and RBT are generally useful for screening for brucellosis especially in developing countries where other tests are cumbersome to perform on a large scale and require special equipment and expertise these tests still have limitations where vaccination or medical records are not available. As a result of these limitations other confirmatory tests like ELISA, CFT, SAT shall be carried out in conjunction with MRT and RBT due to confirm the brucellosis status of cattle in Central Cattle Breeding Station and Dairy Farm, Savar, Dhaka and different Upazilas of Mymensingh, Gaibandha, Rangpur and Jamalpur district. The ELISA is an available assay for use on milk and serum and is very useful where large number of samples require testing. Milk ELISA is used on pooled samples which is more cost effective than testing individual animals. Although these tests may be very expensive they are needed to confirm the brucellosis status of cattle in our study area in order to safeguard there of general public and in particularly that of the people directly involved in the meat inspection, milk collectors and meat milk processing. The aged animals supposed to be more infected because of more contact with infectious agents and sometimes become more susceptible from malnutrition during pregnancy period. It appears that the prevalence of infection in animals more than 5 years of age compared to younger animals and higher prevalence of brucellosis among older cows might be related to maturity with the advancing age. Seropositivity to be considered due to natural infection occurred because vaccination in cows has never been practiced in Bangladesh. The prevalence and severity of disease may vary with the breed, geographic location, types of diagnostic tests, husbandry practice and environmental factors as well as the severity of the organisms. It is important that brucellosis is an important zoonosis and nearly every case of human brucellosis has an animal origin and therefore control is primarily a veterinary responsibility. The sero-prevalence of brucellosis in cows of organized farms and those belonging to domestic holdings of rural areas were studied. Higher occurrences of the diseases were observed in cows of organized farms. Therefore, the MRT should be used for the diagnosis of the brucellosis, especially in 


\section{Conventional PCR based detection of Brucella abortus infected cattle}

Bangladesh. So, if we can diagnosis the milk by MRT it is easy to control infection to human being as well as in animals. The Milk Ring Test is less expensive, it is very easy to do, do not need to any laboratory facilities and do not need expert also. Any person can easily do it in a dairy farm. RBT could sometimes give a false positive result because of S19 vaccination or of false positive serological reactions. Therefore positive reactions should be investigated using suitable confirmatory and/or complementary strategies. False negative reactions occur rarely, mostly due to prozoning and can sometimes be detected by diluting the serum samples or retesting after 4-6 weeks. Nevertheless RBT appears to be adequate as a screening test for detecting infected herds or to guarantee the absence of infection in brucellosis free herds.

Brucellosis has been recognized as an important zoonotic disease as it hampers both animal production and human health. None of the diagnostic test available in Bangladesh are perfect, so screening results needed to be verified by confirmatory test. In this study, milk and serum samples were screened by MRT and RBT and conventional PCR was used as confirmatory test. The MRT is prescribed by OIE for screening of dairy milk samples. It is very easy to perform, cheap and gives a good reflection of serum antibody (Nielsen, 2002; OIE, 2009). The RBT is also used as the standard screening test followed by confirmatory testing as it is very simple, the consumables are cheap, there is a low equipment requirement and the assay is standardized (Nielsen and Ewalt, 2010). This study has screened individual cow's milk by both MRT and RBT in parallel to increase the sensitivity of detecting Brucella shedding cows. It was possible to test 18 samples by conventional PCR, which were positive in both tests. Only $21.43 \%$ of CCBS \&DF samples were PCR positive and none of the nine samples originated from Rangpur, Gaibandha, Jamalpur and Mymensingh districts were positive in PCR. Even being positive in both screening tests, the samples originating from Rangpur, Gaibandha Jamalpur and Mymensingh district were negative in conventional PCR, which may be due to low seroprevalence (1.1-2.1\%) of brucellosis in these areas (Rahman et al., 2011; Dey et al., 2013).Even if the sensitivity and specificity of a test is very high due to low prevalence, the positive predictive value of a test may be very low (Rahman, 2015). It is also possible that some milk samples may contain bacteria below the detection limit and failed to be found as positive. Moreover, it is not possible to detect Brucella DNA by PCR in majority of the MRT positive samples from cows in their chronic phase of the disease (Terzi et al., 2010). On the contrary, the true prevalence and acute infection of brucellosis in CCBSDF were reported to be $20.5 \%$ and $15.6 \%$ respectively (Rahman, 2015). This is very similar to this study finding. As a result more Brucella organisms will be shed in milk in this farm increasing the likelihood of detection in PCR. Obviously identification and culling of acutely infected animals from the population will help to reduce the transmission of the disease in animal populations and thereby it will curb down the zoonotic transmission to humans. From this study finding it can be said that there is more likely chance of shedding Brucella organism with the milk and that may increase of chance likely detection by PCR.

PCR amplification targeting the genus and species-specific genes alkB was performed to confirm the presence of Brucella DNA in milk samples. Detection of an amplicon of $136 \mathrm{bp}$ confirmed the presence of Brucella abortus DNA. Similar finding was reported by Terzi et al. (2010).

Currently veterinary diagnostic laboratories utilize Milk Ring Test (MRT) for diagnosis of bovine milk samples, which indirectly identifies Brucella spp. in the host (Chimana et al., 2010).Just MRT positivity does indicate acute infection. To declare acute infection evidence of Brucella organism or detection of Brucella DNA in animal sample is essential (Bricker, 2002; Gupta et al., 2006; Hamdy and Amin, 2002). Acute infection of brucellosis in dairy cattle can be determined by MRT, RBT and conventional PCR techniques. This finding will help to cull dairy cattle acutely infected with brucellosis having serious public health hazard.

\section{ACKNOWLEDGEMENTS}

The first author is thankful to National Agricultural Technology Programme-1 (NATP-1), DLS, Dhaka for PhD scholarship and Professor Dr. Bahanur Rahman of Department of Microbiology and Hygiene, Bangladesh Agricultural University, Mymensingh for laboratory facilities and technical support.

\section{REFERENCES}

1. Ahasan MS, Rahman MS and Song HJ (2010). A sero-surveillance of Brucella spp. antibodies and individual risk factors of infection in cattle of Bangladesh. Korean Journal of Veterinary Service 33:121-128.

2. Carrera A, Rodriguez LMJ,Sapina AM, Lopez LA and Sacristan AR (2006). Probable transmission of brucellosis by breast milk. Journal of Tropical Pediatrics 52:380-381. 
Sarker and Others

3. Dey SK, Rahman MS, Rima UK, Hossain MZ, Chowdhury GA, Pervin M, Habib MA and Khan MAHNA (2013). Serological and pathological investigation of brucellosis in dairy cows of Mymensingh district Bangladesh. Bangladesh Journal of Veterinary Medicine 11:107-112.

4. Blasco JM, Garin BB, Mann CM, Gerbier G, Fanlo J, Jimenez BMP and Cau C (1994). Efficiency of different Rose Bengal and complement fixation agents for the diagnosis of Brucella melitensis infection in sheep and goats. Veterinary Record 134:415-420.

5. Bricker BJ (2002). PCR as a diagnostic tool for brucellosis. Veterinary Microbiology 90:435-446.

6. Bercovich $\mathrm{Z}$ and Moerman A (1979). Non-specific positive milk ring test(s) in tank milk and Estrumater in the treatment of cattle. Tijdschriftvoor Diergeneeskunde 104:713-716.

7. Chimana HM, Muba JB, Samul KL, Hangombe BM, Munyeme M, Matope G, Phirlam, Skjerve E, Tryland M 2010: A comparative study of the seroprevalence of brucellosis in commercial and small scale mixed dairy beef cattle enterprises of Lusaka province and Chbombo district, Zambia. Tropical Animal Health and Production 42:1541-1545.

8. Erdenebaatar J, Bayarsaikhan B, Yondondorj A, Watarai M, Shirahata T, Jargalsailchan E, Kawamoto K and Makino S (2004). Epidemiological and serological survey of brucellosis in Mongolia by ELISA using sarcosine extracts. Microbiology and Immunology 48:571-577.

9. Gupta, VK, Verma DK, Singh K, Kumari R, Singh SV and Vihan S (2006). Single-step PCR for detection of Brucella melitensis from tissue and blood of goats. Small Ruminant Research 66:169-174.

10. Hamdy MER and Amin AS (2002). Detection of Brucellaspecies in the milk of infected cattle, sheep, goats and camels by PCR. Veterinary Journal 163:299-305.

11. Food and Agriculture Organisation of the United Nations (2004). Bovine brucellosis. Animal health/disease cards.

12. Kato Y, Masuda G, Itoda, Imanura I, Ajisawa A and Negishi A (2007). Brucellosis in a returned traveler and his wife: probable person to person transmission of Brucella melitensis. Journal of Travel Medicine 14:343-345.

13. MacMillan A (1990). Conventional serological test. pp. 153-197. In: Neilsen K, Duncan JR(ed.). Animal brucellosis. CRC Press, Boca Raton.

14. Morgan WJB (1967). The serological diagnosis of bovine brucellosis. Veterinary Record 80: 612-621

15. Nahar A and Ahmed MU (2009). Sero-prevalence study of brucellosis in cattle and contract human in Mymensingh district. Bangladesh Journal of Veterinary Medicine 7:269-274.

16. Nielsen K (2002). Diagnosis of brucellosis by serology. Veterinary Microbiology 90:447-459.

17. Nielsen K and Ewalt D (2010). Bovine Brucellosis. In: Manual of Diagnostic Tests and Vaccines for Terrestrial Animals. OIE: Paris.

18. OIE (2009). Bovine Brucellosis. Manual of standard for diagnostic test. List B disease OIE Terrestrial Manual, Chapter 2, 3, 4.

19. Probert WS, Schrader KN, Khuong NY, Bystrom SL and Graves MH (2004). Real-time multiplex PCR assay for detection of Brucella spp., B.abortus, and B. melitensis. Journal of Clinical Microbiology 42:1290-1293.

20. Rahman MS, Faruk MO, Her M, Kim JY, Kang SI and Jung SC (2011). Prevalence of brucellosis in ruminants in Bangladesh. VeterinariaMedicina 56:379-385.

21. Rahman MS, Sarker MAS, Rahman AKMA, Sarker RR, Melzer F, Sprague LD and Neubauer H (2014). The prevalence of Brucella abortus DNA in seropositive bovine sera in Bangladesh. African Journal of Microbiology Research 8:3856-3860.

22. Rahman AKMA (2015). Epidemiology of brucellosis in humans and domestic ruminants in Bangladesh. PhD thesis, Department of Infectious and Parasitic Diseases, University of Liege, Belgium.

23. Sarker MAS, Rahman MS, Islam MT, Rahman AKMA, Rahman MB, Akter L and Chaki A (2014a). The use of milk ring test for control and eradication of brucellosis in Bangladesh. $2^{\text {nd }}$ International Exhibition on Dairy, Aqua \& Pet Animal. www.iedap.com.144-147.

24. Sarker MAS, Rahman MS, Islam MT, Rahman AKMA, Rahman MB and Rahman MF (2014b). Prevalence of brucellosis in dairy cattle in organized and smallholder farms in some selected areas of Bangladesh. Bangladesh Journal of Veterinary Medicine 12:167-171.

25. Terzi G, Buyuktanir O, Genc O, Gucukoglu A and Yurdusev N (2010). Detection of Brucella antibody and DNA in cow milk by ELISA and PCR methods. The Journal of the Faculty of Veterinary Medicine, University of Kafkas 16:47-52.

26. Tomaso H, Kattar M, Eickhoff M, Werney U, Dahouk SA, Straube E, Neubauer H and Scholz HC (2010). Comparison of commercial DNA preparation kits for the detection of Brucella in tissue using quantitative real-time PCR. BMC Infectious Diseases 10:100. 